\title{
An Algebraic Spectrum Condition
}

\author{
K. KraUS \\ Institut für Theoretische Physik der Universität Marburg \\ Marburg, Germany
}

Received September 24, 1969

\begin{abstract}
A condition, necessary and sufficient for the existence of a vacuum representation with positive energy of the quasilocal algebra, is formulated.

Most postulates of axiomatic quantum field theory can be translated easily into the language of $C^{*}$-algebras [1]. A remarkable exception is the usual spectrum condition. The required algebraic formulation has to assure the existence of a vacuum representation of the quasilocal algebra, for which the energy-momentum spectrum is contained in the future cone $\bar{V}_{+}$. Such a representation will be called a "positive vacuum representation" [4]. Algebraic spectrum conditions have been formulated by Doplicher [2], Montvay [3], and Borchers [4]. In this note, we will propose another condition of this type.

Consider a $C^{*}$-algebra $\mathfrak{A}$, called quasilocal algebra ${ }^{1}$, and a representation $x \rightarrow \alpha_{x}$ of four-dimensional translations $x$ by automorphisms $\alpha_{x}$ of $\mathfrak{A}$. This representation shall be strongly continuous, i.e.,

$$
\lim _{x \rightarrow 0}\left\|\alpha_{x} A-A\right\|=0
$$
\end{abstract}

for any $A \in \mathfrak{A}$. Then $\mathfrak{A}$ contains, as a norm-dense invariant sub-*-algebra $\mathfrak{U}^{(1)}$, the set of all $A \in \mathfrak{U}$ for which

$$
\underset{\tau \rightarrow 0}{\operatorname{norm}-\lim } \frac{1}{\tau}\left(\alpha_{\tau a} A-A\right) \underset{\mathrm{df} .}{=} D_{a} A
$$

exists for all four-vectors $a$ [5].

A positive linear functional $\varphi$ on $\mathfrak{A}$, normalized to $\|\varphi\|=1$, is called a state. Then, for arbitrary state $\varphi$ and $A \in \mathfrak{A}^{(1)}$, the functions

$$
\hat{\varphi}(\tau \mid A, a) \underset{\mathrm{df} .}{=} \varphi\left(A^{*} \alpha_{\tau a} A\right)
$$

are differentiable with respect to $\tau$. Denote by $E_{+}$the set of all states $\varphi$ for which

$$
\left.\frac{1}{i} \frac{d}{d \tau} \hat{\varphi}(\tau \mid A, a)\right|_{\tau=0}=\frac{1}{i} \varphi\left(A^{*} D_{a} A\right) \geqq 0
$$

for all $a \in \bar{V}_{+}$and all $A \in \mathfrak{U}^{(1)}$.

\footnotetext{
${ }^{1}$ The local structure of $\mathfrak{A}$, however, will not be used here.
} 
Since $\mathfrak{U}^{(1)}$ is invariant with respect to the mappings $\alpha_{x}, E_{+}$is invariant with respect to the adjoint mappings $\alpha_{x}^{*}$ of the dual space $\mathfrak{U}^{*}$ of $\mathfrak{A}$, defined by

$$
\left(\alpha_{x}^{*} \varphi\right)(A)=\varphi\left(\alpha_{x} A\right) .
$$

The representation $\alpha_{x}^{*}$ is continuous in the $\mathfrak{A}$ topology of $\mathfrak{U}^{*}$, i.e.,

$$
\lim _{x \rightarrow 0}\left|\left(\alpha_{x}^{*} \varphi\right)(A)-\varphi(A)\right|=0
$$

for all $\varphi \in \mathfrak{U}^{*}, A \in \mathfrak{U}$.

Obviously $E_{+}$is convex. Moreover, it is compact with respect to the $\mathfrak{A}$ topology of $\mathfrak{A}^{*}$. By Ref. [6], Part I, V.4.3. it is sufficient to show that $E_{+}$is closed in this topology, since $E_{+}$is bounded in norm.

For fixed $A \in \mathfrak{Q}^{(1)}$ and fixed $a$, the set $E_{+}(A, a)$ of all states $\varphi$ with

$$
\frac{1}{i} \varphi\left(A^{*} D_{a} A\right) \geqq 0
$$

is closed, since $\frac{1}{i} \varphi\left(A^{*} D_{a} A\right)$ is a continuous function of $\varphi$. By definition, $E_{+}$is the intersection of all sets $E_{+}(A, a)$ with $A \in \mathfrak{O}^{(1)}$ and $a \in \bar{V}_{+}$, and is therefore closed too.

In terms of $E_{+}$an algebraic spectrum condition may then be formulated with the help of the following theorem.

Theorem. $\mathfrak{A}$ possesses a positive vacuum representation if and only if $E_{+}$is not empty.

Proof. If $\pi$ is a positive vacuum representation of $\mathfrak{A}$ with a vacuum vector $\Omega$ and a unitary representation $U(x)=e^{i P x}$ of translations, then the state $\omega$ defined by

$$
\omega(A)=(\Omega, \pi(A) \Omega)
$$

belongs to $E_{+}$. Indeed, for $A \in \mathfrak{A}^{(1)}$ and $a \in \bar{V}_{+}$it follows

$$
\left.\frac{1}{i} \frac{d}{d \tau} \hat{\omega}(\tau \mid A, a)\right|_{\tau=0}=(\pi(A) \Omega, a P \pi(A) \Omega) \geqq 0 .
$$

This proves the necessity of $E_{+} \neq \emptyset$.

In order to prove sufficiency, we note that $\alpha_{x}^{*}$ and $E_{+}$fulfill the assumptions of the Markov-Kakutani fixed-point theorem (Ref. [6], Part I, V.10.6.). Therefore, if $E_{+} \neq \emptyset$, there exists an invariant (vacuum) state

$$
\omega \in E_{+}, \quad \alpha_{x}^{*} \omega=\omega \text { for all } x .
$$

The Gelfand-Segal construction then leads to a representation $\pi$ of $\mathfrak{A}$ in a Hilbert space $\mathscr{H}$, a strongly continuous unitary representation 
$U(x)=e^{i P x}$ of translations with

$$
\pi\left(\alpha_{x} A\right)=U(x) \pi(A) U^{*}(x),
$$

and a cyclic invariant vacuum vector $\Omega \in \mathscr{H}$ with

$$
\omega(A)=(\Omega, \pi(A) \Omega) .
$$

We will show that the self-adjoint energy-momentum operators $P_{v}$ fulfill the spectrum condition or, equivalently, that the self-adjoint operators $a P$ with arbitrary $a \in \bar{V}_{+}$are positive semidefinite.

The vectors $\pi(A) \Omega$ with $A \in \mathfrak{H}^{(1)}$ form a domain $D$ which is dense in $\mathscr{H}$, and is contained in the domain of definition of all operators $a P$. For arbitrary $a \in \bar{V}_{+}$and arbitrary $\Phi=\pi(A) \Omega \in D$,

$$
\begin{aligned}
\left.\frac{1}{i} \frac{d}{d \tau} \hat{\omega}(\tau \mid A, a)\right|_{\tau=0} & =\left.\frac{1}{i} \frac{d}{d \tau}\left(\Omega, \pi\left(A^{*}\right) e^{i \tau a P} \pi(A) \Omega\right)\right|_{\tau=0} \\
& =(\Phi, a P \Phi) \geqq 0
\end{aligned}
$$

since $\omega \in E_{+}$. The restriction $\left.a P\right|_{D}$ of $a P$ to the domain $D$ is therefore a positive semidefinite symmetric operator. Accordingly (Ref. [6], Part II, XII.5.2), it has a positive semidefinite self-adjoint extension $Q_{a}$. Of course, $a P$ itself is also a self-adjoint extension of $\left.a P\right|_{D}$. The proof will be finished by showing that $\left.a P\right|_{D}$ is essentially self-adjoint, which implies $a P=Q_{a}$.

According to Ref. [5], $\mathfrak{U}^{(1)}$ contains a sub-*-algebra $\tilde{\mathfrak{A}}$ of "analytic elements", which is also norm-dense in $\mathfrak{A}$. Using the methods of Ref. [5] one may show that vectors of the form $\pi(A) \Omega$ with $A \in \tilde{\mathcal{U}}$ are analytic vectors for the operators $a P$ with arbitrary $a$. They form a dense subset of $D$. Essential self-adjointness of $\left.a P\right|_{D}$ then follows from a theorem of Nelson [7].

The algebraic spectrum condition proposed here is relatively simple. It has, however, some serious shortcomings. A state $\varphi \in E_{+}$which is not translation invariant has no immediate physical interpretation. In particular, it does not lead to a "positive representation" [4] of $\mathfrak{A}$. Therefore, the set $E_{+}$seems to be of little use if one wants to formulate conditions for the existence of positive representations which do not necessarily have a vacuum.

On the other hand, one may demand the required positive vacuum representation of $\mathfrak{A}$ to be faithful. For this purpose one might try to use, instead of $E_{+}$, the set $E_{+}^{f}$ of states $\varphi \in E_{+}$which lead to faithful representations. However, we can not simply generalize our theorem to this case since we have not been able to show that $E_{+}^{f}$ is compact in some useful topology.

Acknowledgment. A useful comment of Prof. R. Haag is gratefully acknowledged. 


\section{References}

1. Haag, R., Kastler, D.: J. Math. Phys. 5, 848 (1964).

2. Doplicher, S.: Commun. Math. Phys. 1, 1 (1965).

3. Montvay, I.: Nuovo Cimento 40 A, 121 (1965).

4. Borchers, H. J.: On groups of automorphisms with semi-bounded spectrum (Preprint, Göttingen 1969).

5. Kastler, D., Pool, J. C. T., Thue Poulsen, E.: Commun. Math. Phys. 12, 175 (1969).

6. Dunford, N., Schwartz, J. T.: Linear operators. New York: Interscience (Part I: 1958, Part II: 1963).

7. Nelson, E.: Ann. Math. 70, 572 (1959).

K. Kraus

Institut für Theoretische Physik

der Universität

3550 Marburg, Mainzer Gasse 33 